\title{
Prognostic value of exercise pulmonary haemodynamics in pulmonary arterial hypertension
}

\author{
Ari Chaouat ${ }^{1,2}$, Olivier Sitbon ${ }^{3,4}$, Magalie Mercy', Raphaëlle Ponçot-Mongars ${ }^{1}$, \\ Steeve Provencher ${ }^{5}$, Anne Guillaumot ${ }^{1}$, Emmanuel Gomez ${ }^{1}$, \\ Christine Selton-Suty ${ }^{6}$, Pascale Malvestio', Denis Regent', \\ Christophe Paris ${ }^{2,8}$, Philippe Hervé ${ }^{9}$ and François Chabot ${ }^{1,2}$
}

Affiliations: ${ }^{1} \mathrm{CHU}$ Nancy, Pôle des spécialités médicales/Département de Pneumologie, Vandoeuvre-lèsNancy, France. ${ }^{2}$ Université de Lorraine, INGRES, EA 7298, Vandoeuvre-lès-Nancy, France. ${ }^{3} \mathrm{AP}-\mathrm{HP}, \mathrm{CHU}$ de Bicêtre, Service de Pneumologie, Centre de Référence de l'hypertension pulmonaire sévère, DHU TORINO, Le Kremlin-Bicêtre, France. ${ }^{4}$ Université Paris-Sud, INSERM U999, Le Kremlin-Bicêtre, France. ${ }^{5}$ Pulmonary Hypertension Research Group, Centre de recherche de l'Institut Universitaire de Cardiologie et de Pneumologie de Québec, Université Laval, Québec, QC, Canada. ${ }^{6} \mathrm{CHU}$ Nancy, Département de Cardiologie, Vandoeuvre-lès-Nancy, France. ${ }^{7} \mathrm{CHU}$ Nancy, Pôle imagerie/Radiologie, Vandoeuvre-lès-Nancy, France. ${ }^{8} \mathrm{CHU}$ Nancy, Pôle des spécialités médicales/Pathologie Professionnelle, Vandoeuvre-lès-Nancy, France. ${ }^{9}$ Centre Chirurgical Marie-Lannelongue, Service des Explorations Fonctionnelles, Le Plessis-Robinson, France.

Correspondence: Ari Chaouat, Département de Pneumologie, Hôpital de Brabois, CHU Nancy, 54500 Vandoeuvre-lès-Nancy, France. E-mail: a.chaouat@ichu-nancy.fr

ABSTRACT The aim of the study was to investigate the prognostic value of right heart catheterisation variables measured during exercise.

55 incident patients with idiopathic, familial or anorexigen-associated pulmonary arterial hypertension (PAH) underwent right heart catheterisation at rest and during exercise and 6-min walk testing before PAH treatment initiation. Patients were treated according to recommendations within the next 2 weeks. Right heart catheterisation was repeated 3-5 months into the PAH treatment in 20 patients.

Exercise cardiac index decreased gradually as New York Heart Association (NYHA) functional class increased whereas cardiac index at rest was not significantly different across NYHA groups. Baseline 6-min walk distance correlated significantly with exercise and change in cardiac index from rest to exercise $(\mathrm{r}=0.414$ and $\mathrm{r}=0.481$, respectively; $\mathrm{p}<0.01)$. Change in 6 -min walk distance from baseline to $3-5$ months under PAH treatment was highly correlated with change in exercise cardiac index $(r=0.746, p<0.001)$. The most significant baseline covariates associated with survival were change in systolic pulmonary artery pressure from rest to exercise and exercise cardiac index (hazard ratio 0.56 (95\% CI 0.37-0.86) and 0.14 (95\% CI 0.05-0.43), respectively).

Change in pulmonary haemodynamics during exercise is an important tool for assessing disease severity and may help devise optimal treat-to-target strategies.

@ERSpublications

Exercise cardiac index correlated with exercise capacity and was associated with long-term survival in PAH http://ow.ly/vG2bb

This article has supplementary material available from erj.ersjournals.com

Received: Sept 032013 | Accepted after revision: April 042014 | First published online: May 152014

Conflict of interest: Disclosures can be found alongside the online version of this article at erj.ersjournals.com

Copyright @ERS 2014 


\section{Introduction}

According to the Fifth World Symposium on Pulmonary Hypertension held in 2013, group 1 pulmonary hypertension is pulmonary arterial hypertension $(\mathrm{PAH})$, which can be idiopathic, heritable or associated with various conditions [1]. While all PAH subgroups share a number of pathological and clinical features, only idiopathic, heritable, and anorexigen-associated forms of PAH produce a consistent clinical picture in terms of presentation, exercise capacity, pulmonary haemodynamics and survival $[2,3]$.

$\mathrm{PAH}$ is a progressive disease. As pulmonary artery pressure (PAP) and pulmonary vascular resistance (PVR) increase, right ventricle contractility improves initially to preserve cardiac output. When PVR increases further, however, the right ventricle fails, leading to severe functional impairment and ultimately to death [4-6].

Assessing disease severity at diagnosis and its changes under PAH therapy is of paramount importance, as seven drugs targeting three different pharmacological pathways have been approved in North America and Europe and new drugs are expected soon. As a result, determining the best treatment strategy is complex and requires accurate staging and follow-up assessments [7]. In pulmonary hypertension registries [8, 9], cohort studies $[10,11]$ and randomised controlled trials [12], the main prognostic indicators used to date are the New York Heart Association (NYHA) functional class, 6-min walk distance (6MWD), and resting pulmonary haemodynamics evaluated by echocardiography and right heart catheterisation. Currently, resting cardiac output at diagnosis and follow-up evaluation is an important parameter for assessing disease severity and exercise capacity [13].

There is a need to identify variables that predict disease progression early in the course of PAH. These variables must be associated with clinical outcomes and responsive to therapeutic intervention. To enable comparisons with previous studies, they must also correlate with NYHA class, 6MWD and pulmonary haemodynamic parameters.

Among new variables, exercise pulmonary haemodynamics hold promise as major determinants of heart failure in PAH. Exercise stress in patients with PAH tests the ability of the diseased heart to increase its output and the pulmonary arterial system to respond to an increased flow. Previous studies [14, 15] have shown that the effect of prostacyclin analogues on exercise pulmonary haemodynamics was obvious although changes in resting measurements were undetectable.

Here, our objective was to determine whether exercise pulmonary haemodynamics are useful predictors of disease progression in patients with idiopathic, heritable or anorexigen-associated PAH. Parts of the study results have been reported previously at the American Thoracic Society (ATS) conference [16].

\section{Methods}

Additional details on the patients, controls, study design, measurements and statistical analysis are available in the online supplementary material.

\section{Patients}

All patients gave written, informed consent to right heart catheterisation. We studied 43 patients referred to the Nancy University Hospital, Nancy, France, and 12 patients seen at the French national reference centre currently in Le Kremlin-Bicêtre (formerly Clamart), France (fig. 1). These 12 latter patients were included in a previous study [17]. All 55 patients had incident idiopathic, heritable or anorexigen-associated PAH meeting haemodynamic criteria for primary pulmonary hypertension developed at the Second World Symposium, which were similar to current criteria [13]. All patients were evaluated for conditions known to be associated with pulmonary hypertension.

\section{Study design}

Data were collected prospectively at baseline before PAH treatment initiation. Then, all patients started targeted PAH therapy or calcium-channel blocker therapy.

The first follow-up evaluation, which included right heart catheterisation including an exercise test with the same workload used at baseline, was performed in 20 patients 3-5 months after initiating PAH therapy. Subsequently, all the 55 patients underwent clinical evaluation every 3-6 months.

\section{Measurements}

At baseline, all patients underwent right heart catheterisation and 6MWD measurement.

All pulmonary haemodynamic measurements were performed using a Swan-Ganz catheter in the supine position, at rest and during a constant workload exercise. The workload was determined according to the level of exertional dyspnoea and the heart rate during the first minute of the exercise test to allow the patient to exercise in a stable state for $\geqslant 8 \mathrm{~min}$. 


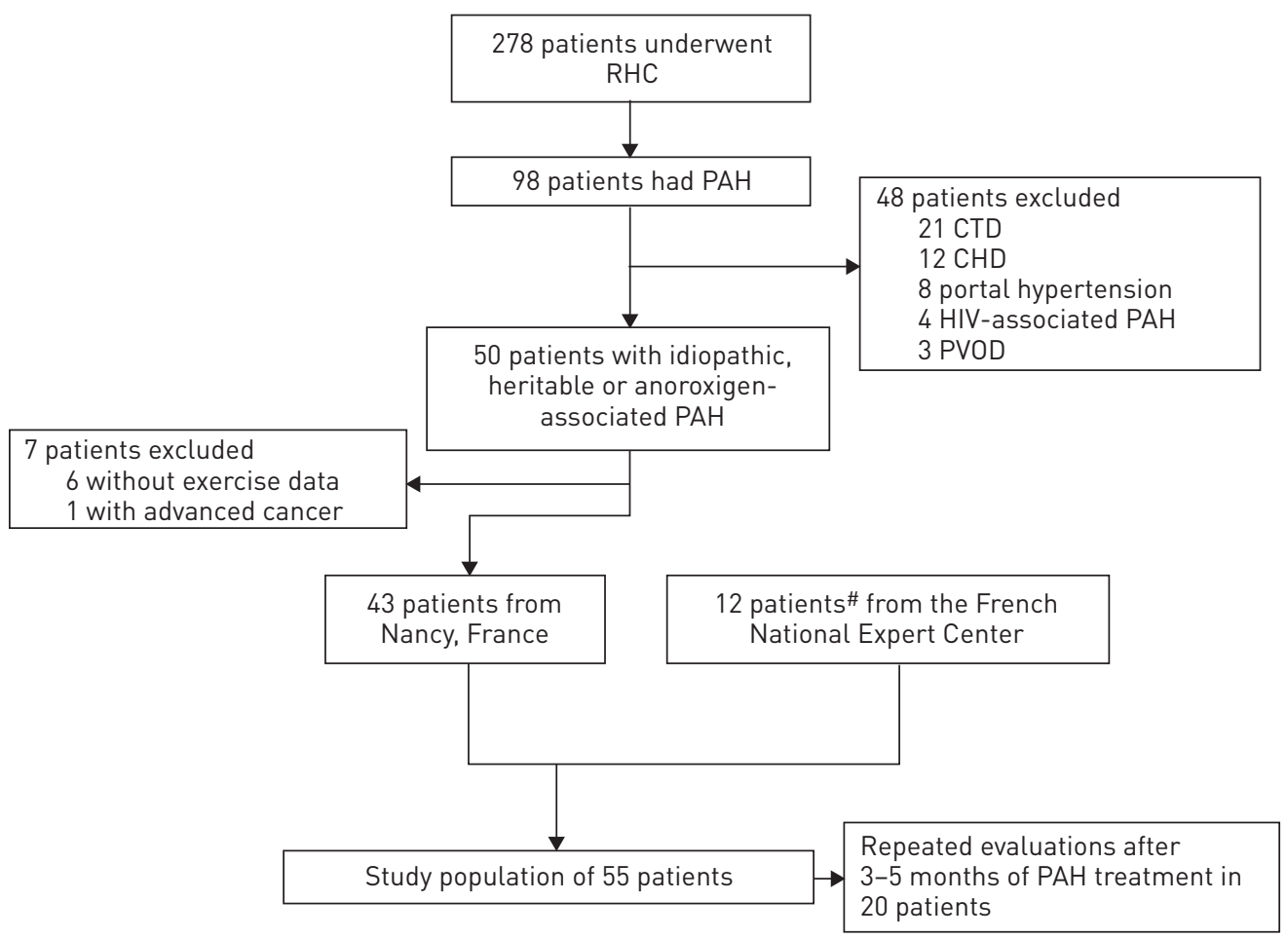

FIGURE 1 Patient flowchart. RHC: right heart catheterisation; PAH: pulmonary arterial hypertension; CTD: connective tissue disease; CHD: congenital heart disease; PVOD: pulmonary veno-occlusive disease. "\#: incident patients with idiopathic, heritable or anorexigen-associated PAH.

The 6MWD was measured in all 55 patients as recommended in ATS guidelines [18].

\section{Statistical analysis}

Differences between independent groups were evaluated using Fisher's exact test, the Mann-Whitney U-test or the Kruskal-Wallis test and correlations by computing Spearman's rho coefficients ( $\mathrm{r}$ ). To identify pulmonary haemodynamic factors independently associated with low $6 \mathrm{MWD}$, we built a multivariate, linear, stepwise regression model with baseline 6MWD as the dependent variable. We compared baseline and follow-up data using the Wilcoxon signed-rank test.

Overall survival was assessed until July 15, 2010, using the Kaplan-Meier method. Predictors of survival available at baseline were identified using Cox proportional-hazards regression models in a univariate analysis followed by forward and backward stepwise analysis. Receiver operating characteristic (ROC) curve analysis of survival at 18 months was used to determine a threshold value for change in cardiac index from rest to exercise as a predictor of survival.

A p-value $<0.05$ was considered significant. Statistical analyses were performed using SPSS Statistics version 19 (IBM, Chicago, IL, USA).

\section{Results}

Patients

Table 1 reports the characteristics of the 55 study patients. Most patients were in NYHA class III-IV but included a wide range of severity according to NYHA functional class assessment and 6MWD. Pulmonary haemodynamic data at rest indicated severe PAH with low values for cardiac index. No technical difficulties or safety issues arose during cardiopulmonary exercise testing.

\section{Stratification by NYHA class}

6MWD was significantly decreased in the NYHA IV group compared with the two less severe NYHA groups (table 2). Right atrial pressure at rest and cardiac index during exercise differed significantly across NYHA groups. Importantly, exercise cardiac index in the NYHA IV group was about half that of the NYHA I/II group and was significantly lower than in the NYHA III group $(\mathrm{p}<0.05)$, whereas cardiac index at rest was not significantly different across NYHA groups. 


\section{TABLE 1 Characteristics of the 55 study patients}

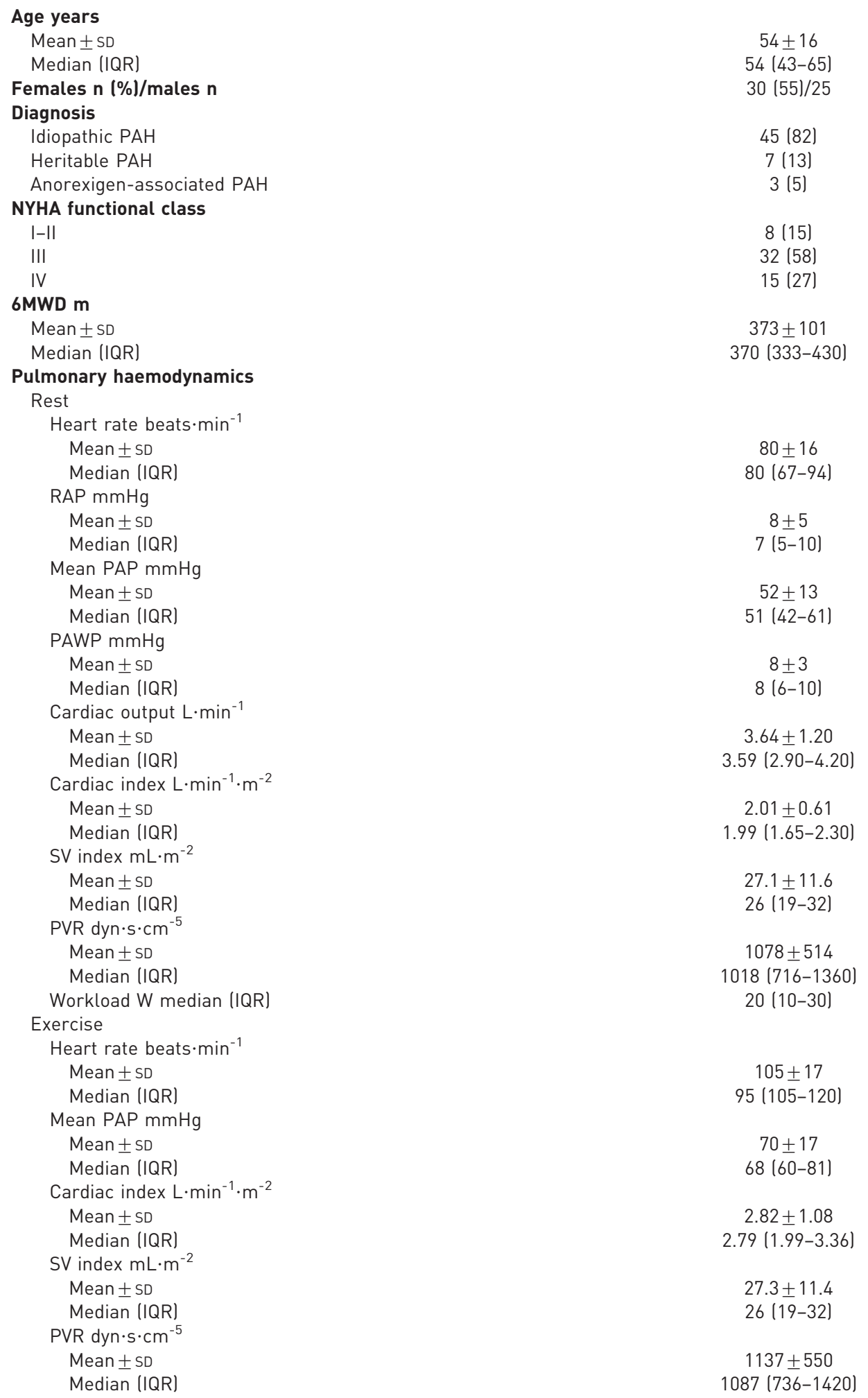

Data are presented as $\mathrm{n}(\%)$, unless otherwise stated. IQR: interquartile range; PAH: pulmonary arterial hypertension; NYHA: New York Heart Association; 6MWD: 6-min walk distance; RAP: right atrial pressure: PAP: pulmonary artery pressure; PAWP: pulmonary artery wedge pressure; SV: stroke volume; PVR: pulmonary vascular resistance. 
TABLE 2 Comparisons of 6-min walk distance (6MWD) and haemodynamic data according to New York Heart Association (NYHA) functional class

NYHA functional class

K-W p-value

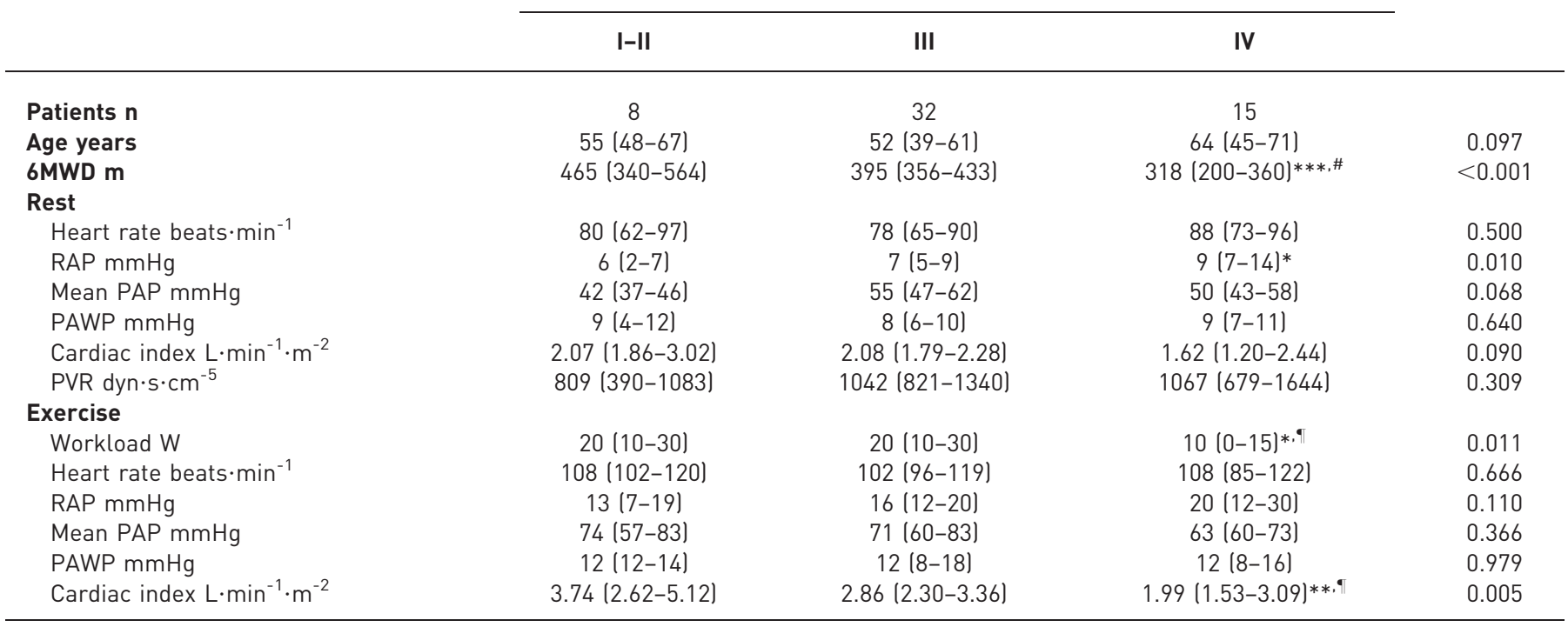

Data are presented as median (interquartile range), unless otherwise stated. K-W: Kruskal-Wallis; 6MWD: 6-min walk distance; RAP: right atrial pressure; PAP: pulmonary artery pressure; PAWP: pulmonary artery wedge pressure; PVR: pulmonary vascular resistance. *: $p<0.05$ by MannWhitney U-test for NYHA IV versus I/II; **: $\mathrm{p}<0.01$ by Mann-Whitney U-test for NYHA IV versus NYHA I/II; ***: $\mathrm{p}<0.001$ by Mann-Whitney U-test for NYHA IV versus I/II; ${ }^{\#}: \mathrm{p}<0.001$ by Mann-Whitney U-test for NYHA IV versus III; ${ }^{\circ}: \mathrm{p}<0.05$ by Mann-Whitney U-test for NYHA IV versus III.

Correlations of baseline variables with baseline 6MWD

Table 3 presents the univariate correlations between baseline 6MWD and other continuous variables at baseline. 6MWD correlated well with exercise cardiac index $(r=0.414, \mathrm{p}=0.002)$ and with the change in cardiac index from rest to exercise $(\mathrm{r}=0.481, \mathrm{p}<0.001)$.

By multiple linear stepwise regression analysis, independent baseline predictors of baseline 6MWD were exercise cardiac index followed by age. No other covariate was selected. The r-values were 0.55 and 0.61 $(\mathrm{p}<0.05$ at each step), respectively (online supplementary table 1$)$.

\section{Changes in 6MWD and pulmonary haemodynamics under treatment}

Table 4 reports the changes in the 20 patients who were re-evaluated after 3-5 months of PAH treatment. These treatments were a calcium-channel blocker $(n=6)$, epoprostenol $(n=2)$, beraprost $(n=2)$, bosentan $(n=8)$, ambrisentan $(n=1)$, and combined bosentan and sildenafil $(n=1)$. Variables that improved with treatment were 6MWD, cardiac index and PVR at rest, and all haemodynamic variables during exercise.

The variable most closely correlated with changes in 6MWD was change in exercise cardiac index from baseline to $3-5$ months under treatment $(r=0.746, p<0.001)$. Figure 2 shows that the $6 \mathrm{MWD}$ changes from baseline to $3-5$ months under PAH therapy were significantly related to changes in cardiac index during exercise but not at rest.

\section{Survival}

At final evaluation or death, all patients were receiving PAH therapy according to recommendations [13]. No patients were lost to follow-up. Three patients received lung transplants $(35,77$ and 83 months after study inclusion). Of the 55 patients, 25 died. Median follow-up among survivors was 67 months (range 5-156 months). Survival after 1, 2 and 3 years was 87\% (95\% CI 78-96\%), 81\% (95\% CI 70-92\%) and 69\% (95\% CI 56-83\%), respectively (fig. 3).

Figure 4 presents the univariate analysis performed to identify baseline variables correlated with survival. The statistically significant predictors of survival were age, NYHA class, 6MWD, right atrial pressure, cardiac index during exercise, and change in systolic PAP and cardiac index from rest to exercise. Survival rates according to the three periods of diagnosis, 2000-2003, 2004-2006 and 2007-2010, were not statistically different $(\mathrm{p}=0.198)$. The multivariate stepwise Cox proportional-hazards analysis showed that 
TABLE 3 Correlations between 6-min walk distance and other continuous variables at baseline

\begin{tabular}{|c|c|c|}
\hline Covariates & $\mathbf{r}$ & p-value \\
\hline Age years & -0.317 & 0.018 \\
\hline \multicolumn{3}{|l|}{ Rest } \\
\hline Heart rate beats $\cdot \mathrm{min}^{-1}$ & -0.058 & 0.678 \\
\hline RAP $\mathrm{mmHg}$ & -0.197 & 0.150 \\
\hline Mean PAP $\mathrm{mmHg}$ & -0.089 & 0.519 \\
\hline Cardiac index $\mathrm{L} \cdot \mathrm{min}^{-1} \cdot \mathrm{m}^{-2}$ & 0.239 & 0.079 \\
\hline PVR dyn $\cdot \mathrm{s} \cdot \mathrm{cm}^{-5}$ & -0.189 & 0.166 \\
\hline \multicolumn{3}{|l|}{ Exercise } \\
\hline Heart rate beats $\cdot \min ^{-1}$ & 0.164 & 0.293 \\
\hline $\mathrm{RAP} \mathrm{mmHg}$ & -0.145 & 0.289 \\
\hline Mean PAP $\mathrm{mmHg}$ & 0.162 & 0.237 \\
\hline Cardiac index $\mathrm{L} \cdot \mathrm{min}^{-1} \cdot \mathrm{m}^{-2}$ & 0.414 & 0.002 \\
\hline Change from rest to exercise of cardiac index $\mathrm{L} \cdot \mathrm{min}^{-1} \cdot \mathrm{m}^{-2}$ & 0.481 & $<0.001$ \\
\hline PVR dyn $\cdot \mathrm{s} \cdot \mathrm{cm}^{-5}$ & -0.329 & 0.020 \\
\hline
\end{tabular}

RAP: right atrial pressure; PAP: pulmonary artery pressure; PVR: pulmonary vascular resistance.

once age was included in the model, only change in cardiac index from rest to exercise further improved the prediction of survival (table 5). None of the other covariates (sex, NYHA class, 6MWD, right atrial pressure, mean PAP, cardiac index, exercise cardiac index or change in systolic PAP from rest to exercise) entered in the equation with forward or backward stepwise methods. Thus, change in cardiac index from rest to exercise was the only independent pulmonary haemodynamic prognostic factor in our population. ROC curve analysis showed a $20 \%$ increase in cardiac index from rest to exercise to be the optimal threshold (70\% sensitivity and $80 \%$ specificity) for determining survival at 18 months (fig. 5 ). All patients with a $\geqslant 50 \%$ increase in cardiac index were alive after 18 months of follow-up.

\section{Discussion}

Our study showed that exercise pulmonary haemodynamic variables at baseline correlated significantly with 6MWD in patients with idiopathic, heritable or anorexigen-associated PAH. In addition, constant workload cardiopulmonary testing during right heart catheterisation was easy to perform and safe. After 3-5 months under PAH therapy, changes in exercise cardiac index correlated well with changes in 6MWD. Finally, baseline exercise cardiac index and changes in baseline cardiac index from rest to exercise were the best independent haemodynamic predictors of survival in our cohort of incident patients.

TABLE 4 6-min walk distance (6MWD) and pulmonary haemodynamics at baseline and after 3-5 months under specific treatment for pulmonary arterial hypertension in the 20 patients who had an exercise testing at the right heart catheterisation re-evaluation

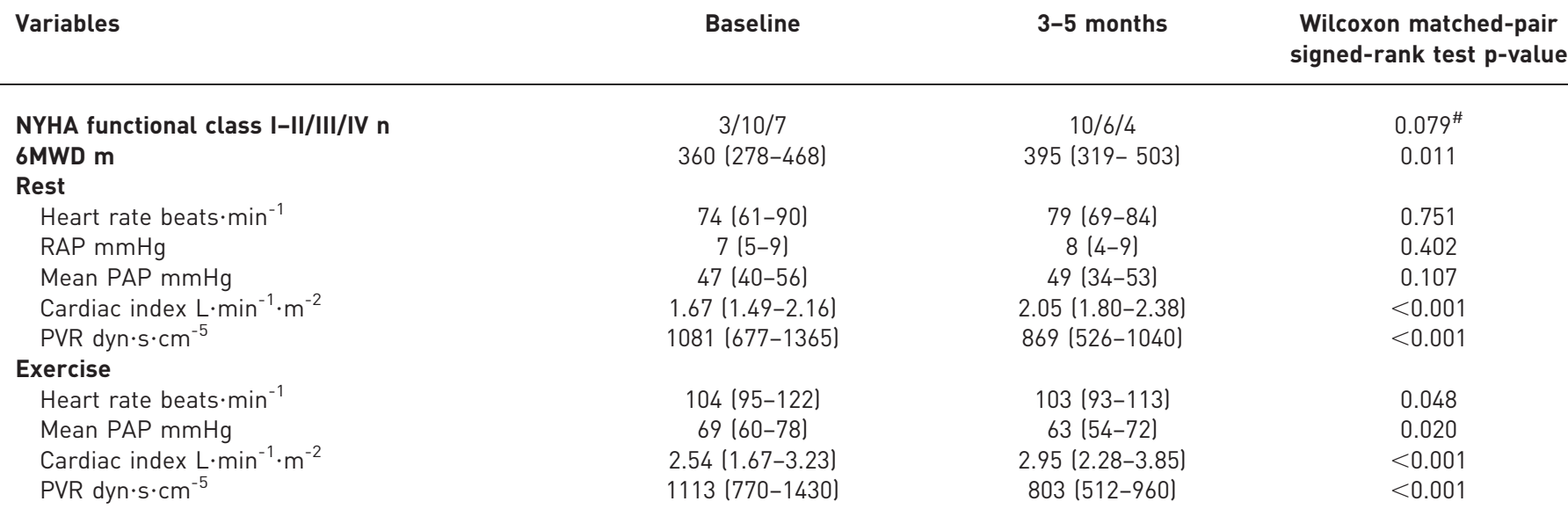

Data are presented as median (interquartile range), unless otherwise stated. NYHA: New York Heart Association; RAP: right atrial pressure; PAP: pulmonary artery pressure; PVR: pulmonary vascular resistance. " : Fisher's exact test. 

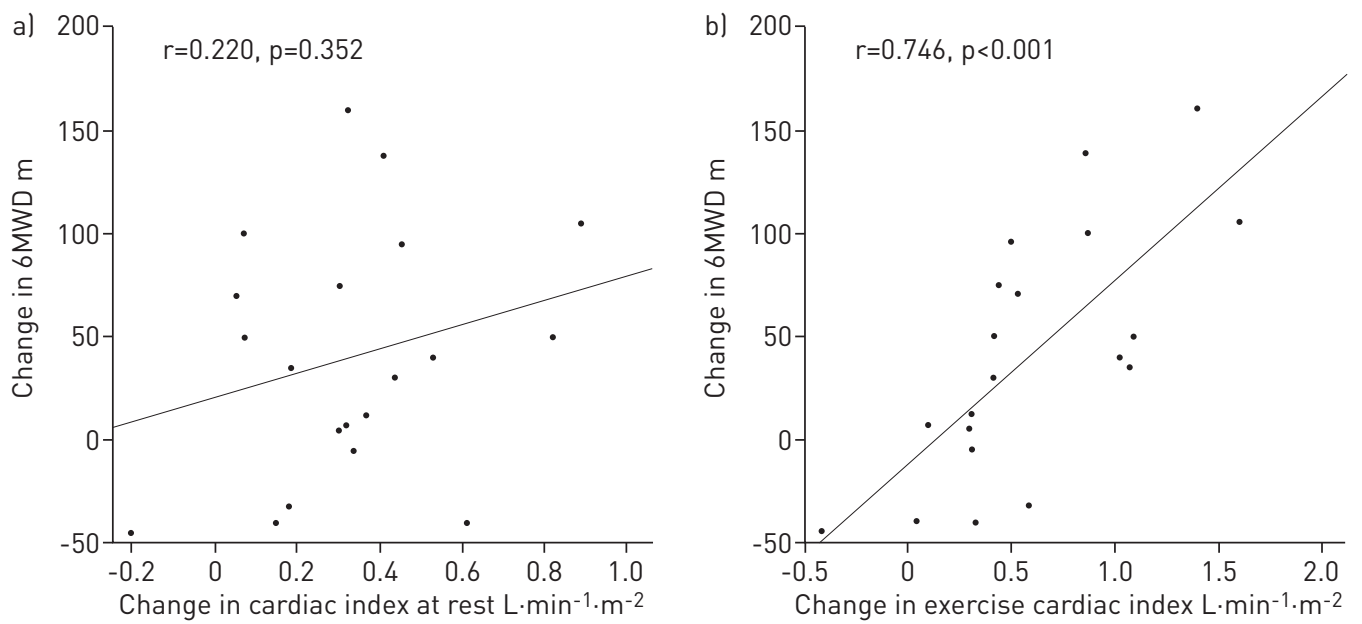

FIGURE 2 Relationship between change in 6-min walk distance (6MWD) and change in cardiac index a) at rest and b) during exercise between baseline and re-evaluation after 3-5 months of treatment in 20 patients with pulmonary arterial hypertension.

The study patients were included prospectively at two centres. All patients were naïve to specific PAH therapy at study inclusion. We included only incident patients, defined as patients diagnosed with PAH within 5 weeks before right heart catheterisation with exercise testing. The age of our patients was consistent with recent case-series studies $[8,9]$. Furthermore, sex ratio, PVR and 3-year mortality were similar to those of incident patients in the French $\mathrm{PAH}$ registry, although a few differences were noted, such as the proportion of NYHA IV patients and the mean 6MWD [9]. Nevertheless, our population seems representative of patients with idiopathic, heritable or anorexigen-associated $\mathrm{PAH}$ seen at expert pulmonary hypertension centres during the study period.

Regarding baseline differences across NYHA groups, exercise cardiac index decreased gradually as the NYHA class increased. A role in this finding for the lower workload in the NYHA IV group is unlikely, as exercise heart rate was similarly increased in all NYHA groups and the low exercise cardiac index in the NYHA IV group was chiefly ascribable to the absence of increase in stroke volume from rest to exercise, a well-known mechanism of inadequate adaptation to exercise in severe PAH [19]. 6MWD in moderate-tosevere $\mathrm{PAH}$ patients is determined by maximal cardiac output $[20,21]$. As in PAH, cardiac output cannot adapt due to an inability to increase heart rate and stroke volume [19,22], it is not surprising to find a significant association between 6MWD and exercise cardiac index in our relatively small population of patients.

Patient recruitment for our study started in 2000. At that time, NYHA III-IV patients received continuous intravenous epoprostenol, and eligible NYHA II-III patients received oral beraprost as participant of a randomised controlled trial published in 2002, followed by an open-label study [23]. Bosentan first became available in France in 2001. Therefore, all patients in the present study received specific PAH therapy at diagnosis and during follow-up. Six patients had acute pulmonary vasoreactivity and received the

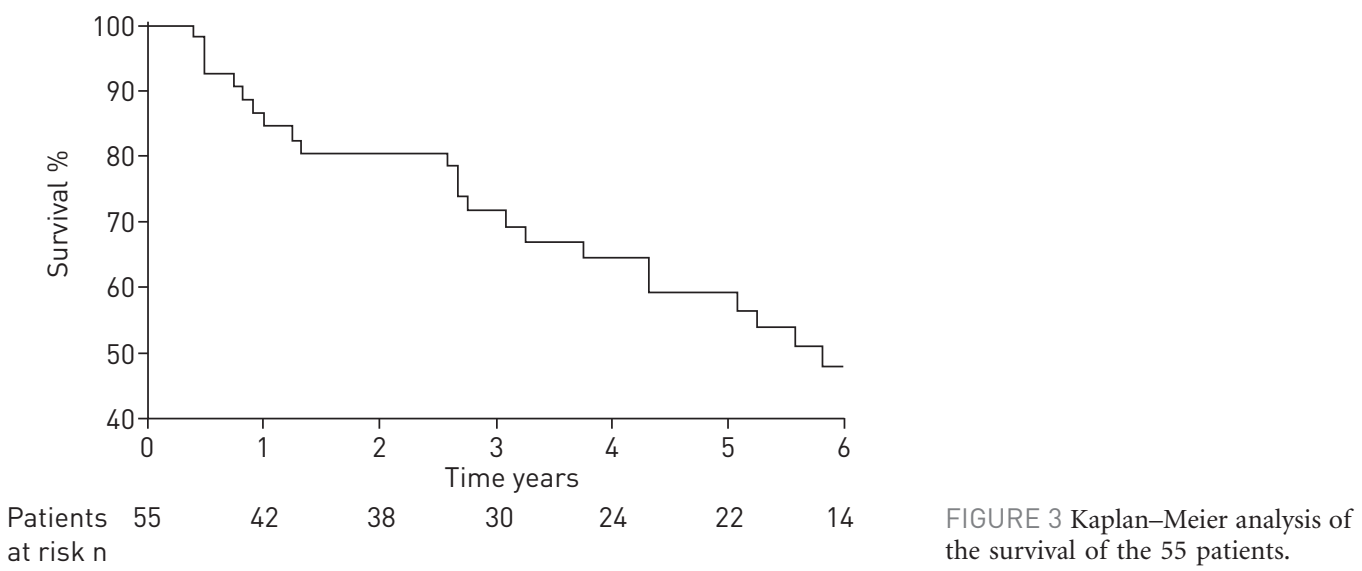


Variable

Age per 10 years

Female sex

NYHA I-II

NYHA III

NYHA IV

6MWD per $30 \mathrm{~m}$

RAP per $\mathrm{mmHg}$

Mean PAP per $10 \mathrm{mmHg}$

Cardiac index at rest per $1 \mathrm{~L} \cdot \mathrm{min}^{-1} \cdot \mathrm{m}^{-2}$

Exercise mean PAP per $10 \mathrm{mmHg}$

Change in systolic PAP per $10 \mathrm{mmHg}$

Exercise cardiac index per $\mathrm{L} \cdot \mathrm{min}^{-1} \cdot \mathrm{m}^{-2}$

Change in cardiac index per L. $\mathrm{min}^{-1} \cdot \mathrm{m}^{-2}$

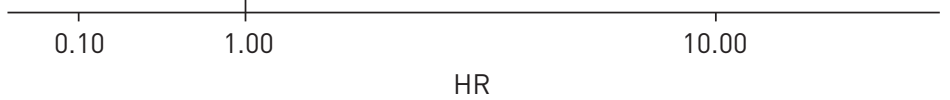

$\mathrm{HR}(95 \% \mathrm{Cl})$

p-value

$1.47(1.10-1.95)$

0.008

$1.19(0.53-2.67)$

0.671

1

$5.22(0.68-40.10)$

0.112

$12.28(1.53-98.43) \quad \mathbf{0 . 0 1 8}$

$0.80(0.70-0.91)$

0.001

$1.11(1.04-1.19)$

0.003

$1.18(0.89-1.56)$

0.259

$0.47(0.20-1.10)$

0.063

$0.91(0.72-1.17)$

0.460

$0.56(0.37-0.86)$

0.008

$0.37(0.21-0.66)$

$<0.001$

$0.14(0.05-0.43)$

$<0.001$

FIGURE 4 Univariate Cox proportional-hazards analysis of survival time according to baseline variables. Whiskers represent $95 \%$ confidence intervals. HR: hazard ratio; NYHA: New York Heart Association; 6MWD: 6-min walk distance; RAP: right atrial pressure; PAP: pulmonary artery pressure.

calcium-channel blocker diltiazem; all these patients underwent follow-up right heart catheterisation and were alive at the end of the study. Two of them lost their responsiveness to calcium-channel blockers; bosentan and bosentan plus sildenafil were added during follow-up. The four long-term calcium-channel responders were kept in the survival analysis to avoid patient-selection bias. This fact may have amplified the relationships between exercise capacity, exercise pulmonary haemodynamics and survival. However, when we excluded the long-term calcium-channel responders, exercise cardiac index remained a good predictor of 6MWD and survival (online supplementary table 2).

We found that exercise cardiac index correlated with established disease severity parameters and significantly predicted survival. Compared with $6 \mathrm{MWD}$, pulmonary haemodynamic values during mild-tomoderate exercise in the supine position may be less affected by peripheral muscle weakness [24], increased dead-space ventilation and dynamic lung hyperinflation [25]. Exercise and change from rest to exercise of cardiac index reflected disease severity more accurately than did cardiac index at rest alone $[14,15,26]$. Change in cardiac index from rest to exercise may be a useful end-point for clinical research and may help to guide treatment decisions in the individual patient. To illustrate the importance of cardiac index during exercise, the ROC curve analysis (fig. 5) showed that patients who were capable of increasing their cardiac index by $\geqslant 50 \%$ during exercise testing at baseline were all alive after 18 months of follow-up even when their cardiac index at rest was low (data not shown). In a previous study, stroke volume at rest was a better prognostic factor than cardiac index [4]. The superiority of stroke volume was explained by the compensatory role of heart rate on the measurement of cardiac index. In our study, the prognostic values of stroke volume at rest and during exercise were similar to those of cardiac index (data not shown). Our findings that changes in cardiac index from rest to exercise at baseline correlate with exercise capacity and survival are in agreement with the very recent published study by GRÜNIG et al. [27]. Our results also showed that an estimate of right ventricular contractile reserve using exercise stress was associated with 6MWD and survival. However, significant differences compared with the study of GRÜNIG et al. [27] may explain why we found that an increase in systolic PAP during exercise was not an independent prognostic

TABLE 5 Multivariate stepwise Cox proportional-hazards analysis of survival time according to selected baseline variables

\begin{tabular}{lcc} 
Variables & Hazard ratio $(95 \% \mathrm{Cl})$ & p-value \\
\hline Age per 10 years & $1.38(1.05-1.82)$ & 0.022 \\
Change in cardiac index per $1 \mathrm{~L} \cdot \mathrm{min}^{-1} \cdot \mathrm{m}^{-2}$ & $0.17(0.06-0.48)$ & 0.001 \\
\hline
\end{tabular}




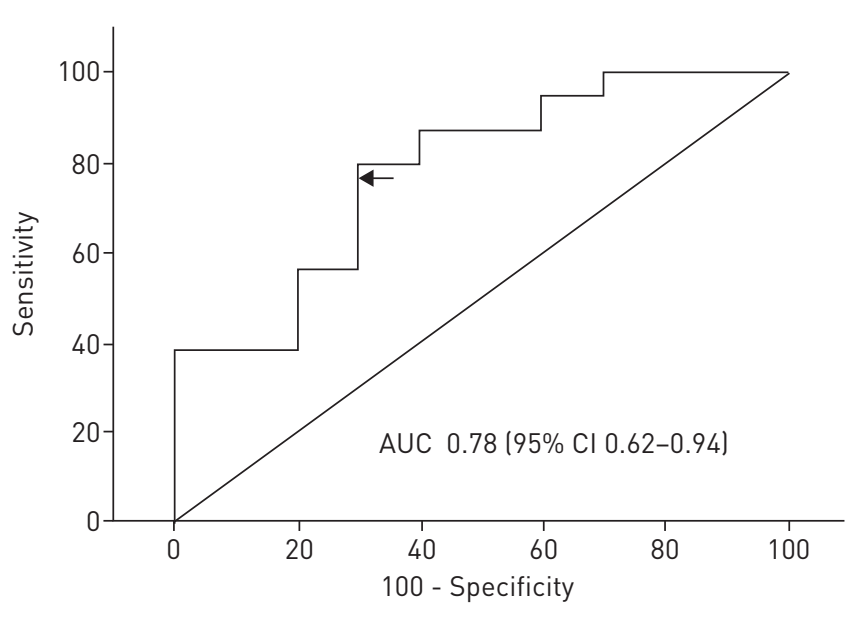

FIGURE 5 Receiver operating characteristic curve analysis of survival at 18 months of follow-up for percentage of change in cardiac index from rest to exercise. The arrow shows that a $20 \%$ increase in cardiac index from rest to exercise had a sensitivity of $70 \%$ and a specificity of $80 \%$ for predicting 18-month survival. AUC: area under the curve.

factor. First, in our study, the workload was lower because of different methodology; second, the study population was much smaller; and last, we were able to measure exercise cardiac index, a different estimate of right ventricular contractile reserve. Stress right heart catheterisation and stress echocardiography may hold promise as prognostic tools.

Our study has several limitations. First, the number of patients is relatively small, including a wide range of severity of the disease. In a larger population, pulmonary haemodynamic values at rest may have significantly predicted exercise capacity and survival $[3,8]$. Due to the relatively small number of patients and a wide range of severity, our findings may not apply to all individuals with idiopathic, heritable or anorexigen-associated PAH. However, our objective was to determine whether exercise pulmonary haemodynamic parameters might help to identify patients at risk and could be used for treatment decisions in the future. Second, the pulmonary haemodynamic parameters measured in our study are less accurate parameters for assessing the exercise response compared with the pressure-flow slope at several workloads [14]. However, with our simpler investigation strategy, the added investigative burden was smaller. Third, multidimensional scores developed in large patient cohorts have been suggested as a good method for outcome prediction [8]. However, in accordance with our study objective, our measurement methods were suitable for an exploratory analysis of haemodynamic during exercise. Fourth, the present study is observational. Although, it must be emphasised that designing a controlled study to determine the role of exercise cardiac index in the outcome of PAH patients would be very challenging. Last, there are seven comparisons of exercise cardiac index and difference in cardiac index from rest to exercise, which could increase by chance a result with $\mathrm{p}<0.05$. It must be emphasised that in these tests, all $\mathrm{p}$-values were $<0.005$, meaning that when applying the Bonferroni correction, the p-value is still $<0.05$.

In conclusion, this study demonstrated that exercise pulmonary haemodynamics could be assessed safely during right heart catheterisation in patients with idiopathic, heritable or anorexigen-associated PAH. The baseline exercise cardiac index and change in cardiac index from rest to exercise predicted exercise capacity and survival. Changes in exercise cardiac index after 3-5 months of specific treatment correlated closely with changes in 6MWD. Our data suggest that exercise cardiac index and change in cardiac index from rest to exercise may hold promise as tools for improving targeted-treatment decisions. These two variables should be further investigated in prospective studies and perhaps in a randomised controlled trial.

\section{Acknowledgements}

We thank Francine Schrijen (CHU Nancy, Pôle des spécialités médicales/Département de Pneumologie, Vandoeuvre-lèsNancy, France) for designing the protocol of cardiopulmonary exercise testing during right heart catheterisation.

\section{References}

1 Simonneau G, Gatzoulis MA, Adatia I, et al. Updated clinical classification of pulmonary hypertension. J Am Coll Cardiol 2013; 62: Suppl., D34-D41.

2 McGoon MD, Benza RL, Escribano-Subias P, et al. Pulmonary arterial hypertension: epidemiology and registries. J Am Coll Cardiol 2013; 62: Suppl., D51-D59.

3 Humbert M, Sitbon O, Yaïci A, et al. Survival in incident and prevalent cohorts of patients with pulmonary arterial hypertension. Eur Respir J 2010; 36: 549-555.

4 van Wolferen SA, Marcus JT, Boonstra A, et al. Prognostic value of right ventricular mass, volume, and function in idiopathic pulmonary arterial hypertension. Eur Heart J 2007; 28: 1250-1257.

5 Haddad F, Doyle R, Murphy DJ, et al. Right ventricular function in cardiovascular disease, part II: pathophysiology, clinical importance, and management of right ventricular failure. Circulation 2008; 117: 1717-1731. 
Champion HC, Michelakis ED, Hassoun PM. Comprehensive invasive and noninvasive approach to the right ventricle-pulmonary circulation unit: state of the art and clinical and research implications. Circulation 2009; 120: 992-1007.

7 Hoeper MM, Bogaard HJ, Condliffe R, et al. Definitions and diagnosis of pulmonary hypertension. J Am Coll Cardiol 2013; 62: Suppl., D42-D50.

8 Benza RL, Miller DP, Gomberg-Maitland M, et al. Predicting survival in pulmonary arterial hypertension: insights from the Registry to Evaluate Early and Long-Term Pulmonary Arterial Hypertension Disease Management (REVEAL). Circulation 2010; 122: 164-172.

9 Humbert M, Sitbon O, Chaouat A, et al. Survival in patients with idiopathic, familial, and anorexigen-associated pulmonary arterial hypertension in the modern management era. Circulation 2010; 122: 156-163.

10 McLaughlin VV, Shillington A, Rich S. Survival in primary pulmonary hypertension: the impact of epoprostenol therapy. Circulation 2002; 106: 1477-1482.

11 Sitbon O, Humbert M, Nunes $\mathrm{H}$, et al. Long-term intravenous epoprostenol infusion in primary pulmonary hypertension: prognostic factors and survival. J Am Coll Cardiol 2002; 40: 780-788.

12 Barst RJ, Rubin LJ, Long WA, et al. A comparison of continuous intravenous epoprostenol (prostacyclin) with conventional therapy for primary pulmonary hypertension. The Primary Pulmonary Hypertension Study Group. N Engl J Med 1996; 334: 296-302.

13 Galiè N, Hoeper MM, Humbert M, et al. Guidelines for the diagnosis and treatment of pulmonary hypertension: the Task Force for the Diagnosis and Treatment of Pulmonary Hypertension of the European Society of Cardiology (ESC) and the European Respiratory Society (ERS), endorsed by the International Society of Heart and Lung Transplantation (ISHLT). Eur Heart J 2009; 30: 2493-2537.

14 Castelain V, Chemla D, Humbert M, et al. Pulmonary artery pressure-flow relations after prostacyclin in primary pulmonary hypertension. Am J Respir Crit Care Med 2002; 165: 338-340.

15 Blumberg FC, Riegger GA, Pfeifer M. Hemodynamic effects of aerosolized iloprost in pulmonary hypertension at rest and during exercise. Chest 2002; 121: 1566-1571.

16 Chaouat A, Poncot-Mongars R, Sitbon O, et al. Impact of exercise pulmonary hemodynamic in idiopathic, heritable and anorexigen-associated pulmonary arterial hypertension. Am J Respir Crit Care Med 2012; 185 : A1887.

17 Provencher S, Hervé P, Sitbon O, et al. Changes in exercise haemodynamics during treatment in pulmonary arterial hypertension. Eur Respir J 2008; 32: 393-398.

18 ATS statement: guidelines for the six-minute walk test. Am J Respir Crit Care Med 2002; 166: 111-117.

19 Groepenhoff H, Holverda S, Marcus JT, et al. Stroke volume response during exercise measured by acetylene uptake and MRI. Physiol Meas 2007; 28: 1-11.

20 Miyamoto S, Nagaya N, Satoh T, et al. Clinical correlates and prognostic significance of six-minute walk test in patients with primary pulmonary hypertension. Comparison with cardiopulmonary exercise testing. Am J Respir Crit Care Med 2000; 161: 487-492.

21 Naeije R. The 6-min walk distance in pulmonary arterial hypertension: "Je t'aime, moi non plus". Chest 2010; 137: $1258-1260$.

22 Provencher S, Chemla D, Hervé P, et al. Heart rate responses during the 6-minute walk test in pulmonary arterial hypertension. Eur Respir J 2006; 27: 114-120.

23 Galiè N, Humbert M, Vachiery JL, et al. Effects of beraprost sodium, an oral prostacyclin analogue, in patients with pulmonary arterial hypertension: a randomized, double-blind, placebo-controlled trial. J Am Coll Cardiol 2002; 39: 1496-1502.

24 Mainguy V, Maltais F, Saey D, et al. Peripheral muscle dysfunction in idiopathic pulmonary arterial hypertension. Thorax 2010; 65: 113-117.

25 Laveneziana P, Garcia G, Joureau B, et al. Dynamic respiratory mechanics and exertional dyspnoea in pulmonary arterial hypertension. Eur Respir J 2013; 41: 578-587.

26 Sun XG, Hansen JE, Oudiz RJ, et al. Exercise pathophysiology in patients with primary pulmonary hypertension. Circulation 2001; 104: 429-435.

27 Grünig E, Tiede H, Enyimayew EO, et al. Assessment and prognostic relevance of right ventricular contractile reserve in patients with severe pulmonary hypertension. Circulation 2013; 128: 2005-2015. 